\title{
A new species of Pareas (Squamata, Pareidae) from southern Vietnam
}

\author{
Duong T. T. Le ${ }^{1,2}$, Thinh G. Tran ${ }^{1}$, Huy D. Hoang ${ }^{1,2}$, Bryan L. Stuart ${ }^{3}$ \\ 1 Department of Ecology and Evolutionary Biology, Faculty of Biology and Biotechnology, University of Science, Ho Chi Minh City, 227 Nguyen \\ Van Cu Street, District 5, Ho Chi Minh City, Vietnam \\ 2 Vietnam National University, Ho Chi Minh City, Linh Trung Ward, Thu Duc, Ho Chi Minh City, Vietnam \\ 3 North Carolina Museum of Natural Sciences, 11 West Jones Street, Raleigh, North Carolina 27601, USA
}

http://zoobank.org/C1F01E38-E26F-446C-985F-96B76E68CA7C

Corresponding author: Duong T. T. Le (lttduong@hcmus.edu.vn)

\begin{tabular}{l|lllll}
\hline Academic editor Uwe Fritz & Received 23 June 2021 $\quad$ Accepted 27 July $2021 \quad \mid$ Published 5 August 2021 \\
\hline
\end{tabular}

Citation: Le DTT, Tran TG, Hoang HD, Stuart BL (2021) A new species of Pareas (Squamata, Pareidae) from southern Vietnam. Vertebrate Zoology 71: 439-451. https://doi.org/10.3897/vz.71.e70438

\begin{abstract}
We describe a new species of pareid snake from the Di Linh Plateau in Lam Dong Province of southern Vietnam based on morphological and molecular evidence. Pareas temporalis sp. nov. is distinguished from its congeners by having the combination of yellow-brown body colouration; hexagonal-shaped frontal, with lateral sides parallel to the body axis; $16-17$ temporals, with 4-5 anterior temporals; loreal and prefrontal not contacting eye; 2-3 preoculars; two suboculars; 2-3 postoculars; 8-9 supralabials; 8-9 infralabials; $15-15-15$ dorsal scale rows, all keeled, three vertebral scale rows enlarged; 191 ( +1 preventral) ventrals, smooth; 92 subcaudals, all divided; undivided anal scale; two postocular stripes; and a solid dark brown vertebral stripe extending from rear of nuchal collar along the entire length of body and tail. Phylogenetic analyses of mitochondrial DNA data recovered the new species to be nested within the P. carinatus complex and to be the sister taxon to P. nuchalis from Borneo.
\end{abstract}

\section{Keywords}

Di Linh Plateau, integrative taxonomy, Pareatinae, Pareas menglaensis, Southeast Asia

\section{Introduction}

The genus Pareas is the most species-diverse genus in the subfamily Pareatinae and is distributed throughout tropical and subtropical parts of Asia (Rao and Yang 1992). It differs from other pareid genera by having 15 rows of dorsal scales at midbody, divided subcaudals, suboculars, supralabials not touching the eye, no anterior single inframaxillary, and three pairs of inframaxillaries (Grossmann and Tillack 2003). The monophyly of Pareas with respect to other pareid genera has been recovered with statistical support in some studies (e.g., Ding et al. 2020; Vogel et al. 2020), but not in others (e.g., Guo et al. 2011; Pyron et al. 2013; You et al. 2015; Figueroa et al. 2016; Liu and Rao 2021).

After 77 years without any descriptions of new species in the genus (from 1937 to 2014), seven new species have been described since 2015, mostly based on integrative taxonomic approaches incorporating molecular analyses and morphological comparisons (Liu and Rao 2021; Uetz 
et al. 2021; Vogel et al. 2021). These integrative taxonomic approaches have also clarified some taxonomic uncertainties in previously described species. For example, recent studies have shown $P$. macularius Theobald, 1868 to be a species distinct from $P$. margaritophorus (Jan, 1866) (e.g., Hauser 2017; Suntrarachun et al. 2020; Vogel et al. 2020) following their synonymy by Huang (2004). To the contrary, Liu and Rao (2021) considered $P$. mengziensis, a new species very recently described by Wang et al. (2020), to be conspecific with P. niger Pope, 1928 based on molecular and morphological data.

During field work in the Di Linh Plateau at the southernmost tip of the Truong Son mountain range in Vietnam, we discovered a single specimen of Pareas that differed in morphological and molecular data from all members of the genus. Based on these corroborated lines of evidence, we describe this species as new.

\section{Methods}

Fieldwork was carried out by DTTL and TGT in Doan Ket Commune, Da Huoai District, Lam Dong Province, Vietnam, in July 2020. The specimen was stored in 75\% ethanol after preserving liver in 99\% ethanol. Specimens and tissue samples were deposited at the Lab of Zoology, University of Science, Ho Chi Minh City (UNS).

\section{Morphometrics}

Measurements of the single Di Linh specimen were taken to the nearest $0.1 \mathrm{~mm}$ with dial calipers. Paired meristic characters are given as left/right. Measurements and meristic counts were taken following Wang et al. (2020). Morphological measurements (all in mm) and scale counts included snout-vent length (SVL); tail length $(\mathrm{TaL})$; total length (TL); relative tail length (TaL/ TL); number of dorsal scales counted at approximately one head length behind the head, midbody, and one head length before vent; ventral scales; subcaudal scales; supralabials; infralabials; number of keeled dorsal scale rows at midbody; anterior temporals; posterior temporals; preoculars; and postoculars.

Comparative data for other species were taken from relevant publications (Boulenger 1900; Boulenger 1914; Malkmus et al. 2002; Guo and Deng 2009; Stuebing et al. 2014; Vogel 2015; You et al. 2015; Bhosale et al. 2020; Ding et al. 2020; Suntrarachun et al. 2020; Vogel et al. 2020; Wang et al. 2020; Liu and Rao 2021; Vogel et al. 2021).

\section{Molecular sequencing}

Total DNA was extracted from liver preserved in $99 \%$ ethanol using aTopPURE genomic DNA extraction kit (ABT Biological solutions company limited, Vietnam).
Two mitochondrial gene fragments, cytochrome $b$ (cyt $b$ ) and NADH dehydrogenase subunit 4 and its flanking tRNAs (ND4), were amplified by the polymerase chain reaction (PCR) using the primers L14910/H16064 (Burbrink et al. 2000) and ND4/Leu (Arèvalo et al. 1994). PCR conditions for cyt $b$ were denaturation at $94^{\circ} \mathrm{C}$ for seven minutes, followed by 40 cycles at $94^{\circ} \mathrm{C}$ for $40 \mathrm{sec}-$ onds, $46^{\circ} \mathrm{C}$ for 30 seconds and $72^{\circ} \mathrm{C}$ for one minute, with a final extension step at $72^{\circ} \mathrm{C}$ for seven minutes. PCR conditions for ND4 were denaturation at $92^{\circ} \mathrm{C}$ for three minutes, followed by 35 cycles at $92^{\circ} \mathrm{C}$ for 45 seconds, $40^{\circ} \mathrm{C}$ for two minutes and $70^{\circ} \mathrm{C}$ for 1.5 minutes, with a final extension step at $70^{\circ} \mathrm{C}$ for five minutes. Sequences were edited using Chromas 2.6.6 (Technelysium Pty. Ltd.) and Geneious Prime 2021.1 (Biomatters Ltd.).

In addition, a 1,071 bp fragment of cyt $b$ of $P$. nuchalis (FK 2626; Table 1) was provided to the authors after being obtained as bycatch during a phylogenomic analysis by D. L. Rabosky et al. (in prep.). Newly generated sequences were deposited in GenBank under accession numbers MZ603792- MZ603794 (Table 1).

\section{Phylogenetic analysis}

Homologous sequences of all currently recognized species of Pareas, two representatives of each of the pareid genera Aplopeltura, Asthenodipsas, and Xylophis, and the xenodermatid outgroup Achalinus rufescens (following Figueroa et al. 2016, Deepak et al. 2018, Li et al. 2020) were downloaded from GenBank (Table 1). Newly generated and downloaded sequences were aligned using the default parameters in the MAFFT 7.45 alignment algorithm (Katoh and Standley 2013) implemented in Geneious Prime 2021.1.1 (Biomatters Ltd.). Alignments were visually checked to ensure that insertion-deletions did not disrupt translation of coding regions. The dataset was partitioned by tRNA and codon positions of cyt $b$ and ND4 for a total of seven partitions. The best-fit partitioning scheme and models of sequence evolution were selected using the Akaike Information Criterion (AICc) in Partition Finder 2 (Lanfear et al. 2017). Four partitions were selected with the models TVM $+\mathrm{I}+\mathrm{G}$ for tRNA, and cyt $b$ and ND4 first codon positions; TVM $+\mathrm{I}+\mathrm{G}$ for cyt $b$ and ND4 second codon positions; $\mathrm{TRN}+\mathrm{I}+\mathrm{G}$ for cyt $b$ third codon position; and TIM+I+G for ND4 third codon position.

Bayesian inference (BI) was performed on the partitioned dataset using MrBayes 3.2.7a (Ronquist et al. 2012) on the Cyber infrastructure for Phylogenetic Research (CIPRES) Science Gateway version 3.3 (Miller et al. 2010). In each of four independent analyses, four chains were run for 20 million generations using the default priors, trees were sampled every 4,000 generations, and the first $25 \%$ of trees were discarded as 'burn-in'. The resulting trace plots were viewed using Tracer v.1.7 (Rambaut et al. 2018). A 50\% majority-rule consensus of the post burn-in trees was constructed to calculate the posterior probabilities of nodes. Maximum likelihood (ML) analysis was performed on the partitioned dataset 
Table 1. Samples used in the molecular phylogenetic analyses. Institutional and collector abbreviations of vouchers are defined in the source publications.

\begin{tabular}{|c|c|c|c|c|c|}
\hline Taxon & Voucher & Locality & cytb & ND4 & Sources \\
\hline Achalinus rufescens & HS 14023 & China, Anhui, Qimen & KT897595 & KT897595 & Zhang et al. (2017) \\
\hline Aplopeltura boa & KIZ 011963 & Malaysia & JF827673 & JF827650 & Guo et al. (2011) \\
\hline Ap. boa & LSUHC 7248 & Malaysia, Sabah, Sepilok & KC916746 & - & Loredo et al. (2013) \\
\hline Ap. boa & UMMZ 201905 & Brunei, Belait & - & ABU49312 & Kraus and Brown (1998) \\
\hline Asthenodipsas laevis & FMNH 241296 & Malaysia, Sabah, Lahad Datu & KX660468 & KX660596 & Figueroa et al. (2016) \\
\hline As. laevis & FMNH 273617 & Malaysia, Sarawak, Bintulu & KX660469 & KX660597 & $\begin{array}{l}\text { Figueroa et al. (2016), } \\
\text { Quah et al. (2019) }\end{array}$ \\
\hline Pareas andersonii & CAS 235359 & Myanmar, Chin, Mt. Natmataung & MT968772 & MW287040 & $\begin{array}{l}\text { Ding et al. (2020), } \\
\text { Vogel et al. (2020) }\end{array}$ \\
\hline P. atayal & NMNS 05594 & China, Taiwan, Yilan, Beiheng & KJ642124 & MW287041 & $\begin{array}{l}\text { You et al. (2015), } \\
\text { Ding et al. (2020) }\end{array}$ \\
\hline P. atayal & HC 000618 & China, Taiwan, Yilan & JF827685 & JF827662 & Guo et al. (2011) \\
\hline P. atayal & HC 000628 & China, Taiwan, Taoyuan & JF827686 & JF827663 & Guo et al. (2011) \\
\hline P. boulengeri & None & China, Anhui, Qimen, Huangjialing & MN866896 & MN866896 & Huang et al. (2020) \\
\hline P. boulengeri & KIZ 09965 & China, Hubei, Enshi & JF827678 & JF827655 & Guo et al. (2011) \\
\hline P. boulengeri & GP 2923 & China, Guizhou, Jiangkou & MK135090 & MK805355 & Wang et al. (2020) \\
\hline P. boulengeri & GP 207 & China, Sichuan, Anxian & MK135091 & MK805356 & Wang et al. (2020) \\
\hline P. boulengeri & GP $3095=$ YBU 13323A & China, Hubei, Wufeng & MK135092 & MK805357 & Wang et al. (2020) \\
\hline P. boulengeri & GP 4716 & Yidu, Hubei, China & MK135093 & MK805358 & Wang et al. (2020) \\
\hline P. boulengeri & GP 3428 & China, Anhui, Yixian & MK135094 & MK805359 & Wang et al. (2020) \\
\hline P. boulengeri & GP $4827=$ YBU 17155 & China, Zhejiang, Chunan & MK135095 & MK805360 & Wang et al. (2020) \\
\hline P. boulengeri & GP $4886=$ YBU 17245 & China, Zhejiang, Chunan & MK135096 & MK805361 & Wang et al. (2020) \\
\hline P. carinatus & DL 2008-S039 & Malaysia & JF827677 & JF827653 & Guo et al. (2011) \\
\hline P. carinatus & GP 1079 & Malaysia, Kuala Lumpur & MK135110 & MK805375 & Wang et al. (2020) \\
\hline P. carinatus & GP $5131=$ KIZ 011972 & Malaysia, Kuala Lumpur & MK135111 & MK805376 & Wang et al. (2020) \\
\hline P. carinatus & GP $5129=$ KIZ 011970 & Malaysia, Kuala Lumpur & MK135112 & MK805377 & Wang et al. (2020) \\
\hline P. carinatus & CAS 247982 & Myanmar, Tanintharyi, Yaephyu & MT968778 & - & Vogel et al. (2020) \\
\hline P. carinatus & LSUHC 10604 & Malaysia, Kedah, Sungai Sedim & KC916748 & - & Loredo et al. (2013) \\
\hline P. chinensis & CIB 098269 & China, Sichuan, Tianquan & JF827691 & JF827668 & Guo et al. (2011) \\
\hline P. chinensis & GP 2196 & China, Sichuan, Junlian & MK135088 & MK805353 & Wang et al. (2020) \\
\hline P. chinensis & GP 2383 & China, Sichuan, Hongya & MK135089 & MK805354 & Wang et al. (2020) \\
\hline P. formosensis & GP $2146=$ YBU 12015 & China, Hainan & MK135068 & MK805333 & Wang et al. (2020) \\
\hline P. formosensis & GP 2164 & China, Hainan & MK135069 & MK805334 & Wang et al. (2020) \\
\hline P. formosensis & GP 2165 & China, Hainan & MK135070 & MK805335 & Wang et al. (2020) \\
\hline P. formosensis & GP $2170=$ YBU 12032 & China, Hainan & MK135071 & MK805336 & Wang et al. (2020) \\
\hline P. formosensis & GP 4581 & China, Zhejiang, Jingning & MK135072 & MK805337 & Wang et al. (2020) \\
\hline P. formosensis & GP $4659=$ YBU 17029 & China, Hainan & MK135073 & MK805338 & Wang et al. (2020) \\
\hline P. formosensis & GP $2332=$ YBU 12090 & China, Guizhou, Leishan & MK135074 & MK805339 & Wang et al. (2020) \\
\hline P. formosensis & GP $2384=$ YBU 12115 & China, Guizhou, Rongjiang & MK135075 & MK805340 & Wang et al. (2020) \\
\hline P. formosensis & GP $3911=$ YBU 14508 & China, Guangxi & MK135076 & MK805341 & Wang et al. (2020) \\
\hline P. formosensis & GP 3696 & China, Jiangxi, Yanshan & MH046857 & MK805382 & Wang et al. (2020) \\
\hline P. formosensis & GP 3808 & China, Jiangxi, Yanshan & MH046858 & MK805383 & Wang et al. (2020) \\
\hline P. formosensis & GP $3859=$ YBU 14573 & China, Jiangxi, Yanshan & MH046859 & MK805384 & Wang et al. (2020) \\
\hline P. formosensis & NMNH 05637 & China, Taiwan, Nantou, Xitou & MW287060 & MW287042 & Ding et al. (2020) \\
\hline P. formosensis & H26-HAM01 & China, Guangdong & MW287061 & MW287043 & Ding et al. (2020) \\
\hline P. formosensis & ZMMU R-16684 & Vietnam, Cao Bang, Phia Bac & MW287062 & MW287044 & Ding et al. (2020) \\
\hline P. formosensis & ZMMU NAP-08868 & Vietnam, Quang Nam, Song Thanh & MW287063 & MW287045 & Ding et al. (2020) \\
\hline P. formosensis & ZMMU R-13709 & Vietnam, Lam Dong, Bidoup - Nui Ba & MW287064 & MW287046 & Ding et al. (2020) \\
\hline P. formosensis & ZMMU R-14072 & Vietnam, Dak Lak, Chu Yang Sin & MW287065 & MW287047 & Ding et al. (2020) \\
\hline P. formosensis & ZMMU R-16333 & Vietnam, Gia Lai, Kon Chu Rang & MW287066 & MW287048 & Ding et al. (2020) \\
\hline P. geminatus & $\begin{array}{l}\text { ZMMU NAP-09280 = } \\
\text { R-16695 }\end{array}$ & Laos, Xaisomboun, Long Tien & MW287073 & MW287049 & Ding et al. (2020) \\
\hline P. geminatus & ZMMU R-16478 & Thailand, Chiang Mai, Doi Inthanon & MW287074 & MW287050 & Ding et al. (2020) \\
\hline P. geminatus & ZMMU R-16477 & Thailand, Chiang Mai, Mae Kampong & MW287075 & MW287051 & Ding et al. (2020) \\
\hline P. geminatus & AUP-00176 & Thailand, Chiang Mai, Doi Inthanon & MW287076 & MW287052 & Ding et al. (2020) \\
\hline P. hamptoni & GP $5127=$ YPX 18219 & Myanmar, Kachin & MK135077 & MK805342 & Wang et al. (2020) \\
\hline
\end{tabular}




\begin{tabular}{|c|c|c|c|c|c|}
\hline Taxon & Voucher & Locality & cytb & ND4 & Sources \\
\hline P. hamptoni & GP $5128=$ YPX 18604 & Myanmar, Kachin & MK135078 & MK805343 & Wang et al. (2020) \\
\hline P. hamptoni & ZMMU NAP-09087 & Vietnam, Lao Cai, Bat Xat & MW287078 & MW287054 & Ding et al. (2020) \\
\hline P. hamptoni & ZMMU NAP-09088 & Vietnam, Lao Cai, Bat Xat & MW287079 & MW287053 & Ding et al. (2020) \\
\hline P. iwasakii & I03-ISG1 & Japan, S. Ryukyu, Ishigaki & KJ642158 & - & You et al. (2015) \\
\hline P. kaduri & BNHS 3574 & India, Arunachal Pradesh, Lohit & MT188734 & - & Bhosale et al. (2021) \\
\hline P. komaii & HC 000669 & China, Taiwan, Taitung, Lijia & JF827687 & JF827664 & Guo et al. (2011) \\
\hline P. komaii & NMNS 05625 & China, Taiwan, Hualien & KJ642189 & MW287055 & $\begin{array}{l}\text { You et al. (2015), } \\
\text { Ding et al. (2020) }\end{array}$ \\
\hline P. komaii & NMNS 05618 & China, Taiwan, Taitung, Lijia & KJ642185 & MW287056 & $\begin{array}{l}\text { You et al. (2015), } \\
\text { Ding et al. (2020) }\end{array}$ \\
\hline P. macularius & GP 815 & China, Hainan & MK135101 & MK805366 & Wang et al. (2020) \\
\hline P. macularius & GP 2110 & China, Hainan & MK135102 & MK805367 & Wang et al. (2020) \\
\hline P. macularius & GP $2147=$ YBU 12016 & China, Hainan & MK135103 & MK805368 & Wang et al. (2020) \\
\hline P. macularius & GP $4660=$ YBU 17030 & China, Hainan & MK135104 & MK805369 & Wang et al. (2020) \\
\hline P. macularius & GP $4715=$ YBU 17078 & China, Yunnan, Jingdong & MK135105 & MK805370 & Wang et al. (2020) \\
\hline P. macularius & GP $4699=$ YBU 17062 & China, Yunnan, Jingdong & MK135106 & MK805371 & Wang et al. (2020) \\
\hline P. macularius & ZMMU R-16629 & Myanmar, Sagaing, Ban Mauk & MT968771 & MW287057 & $\begin{array}{l}\text { Ding et al. (2020), } \\
\text { Vogel et al. (2020) }\end{array}$ \\
\hline P. margaritophorus & GP $4410=$ YBU 16061 & China, Guangxi, Cangwu & MK135097 & MK805362 & Wang et al. (2020) \\
\hline P. margaritophorus & GP $4837=$ YBU 17164 & China, Guangxi, Cangwu & MK135098 & MK805363 & Wang et al. (2020) \\
\hline P. margaritophorus & GP 4437 & China, Guangxi, Cangwu & MK135099 & MK805364 & Wang et al. (2020) \\
\hline P. margaritophorus & GP $4465=$ YBU 16095 & China, Guangxi, Cangwu & MK135100 & MK805365 & Wang et al. (2020) \\
\hline P. margaritophorus & M01 & Vietnam, Binh Phuoc, Bu Gia Map & KJ642195 & MW287058 & $\begin{array}{l}\text { You et al. (2015), } \\
\text { Ding et al. (2020) }\end{array}$ \\
\hline P. menglaensis & GP 1292 & China, Yunnan, Mengla & MK135113 & MK805378 & Wang et al. (2020) \\
\hline P. menglaensis & GP $3356=$ YBU 14124 & China, Yunnan, Mengla & MK135114 & MK805379 & Wang et al. (2020) \\
\hline P. menglaensis & GP $3376=$ YBU 14141 & China, Yunnan, Mengla & MK135115 & MK805380 & Wang et al. (2020) \\
\hline P. menglaensis & GP $3377=$ YBU 14142 & China, Yunnan, Mengla & MK135116 & MK805381 & Wang et al. (2020) \\
\hline P. modestus & MZMU 1293 & India, Mizoram, Aizawl, Tanhril & MT968773 & - & Vogel et al. (2020) \\
\hline P. monticola & ZMMU R-16631 & Myanmar, Sagaing, Ban Mauk & MW438296 & MW438301 & Vogel et al. (2021) \\
\hline P. monticola & ADR 507 & India, Assam, Orang & MN970038 & MN970043 & Deepak et al. (2020) \\
\hline P. monticola & GP 2027 & China, Xizang, Motuo & MK135107 & MK805372 & Wang et al. (2020) \\
\hline P. monticola & GP $5132=$ KIZ 047036 & China, Yunnan, Pingbian & MK135108 & MK805373 & Wang et al. (2020) \\
\hline P. monticola & GP $5133=$ KIZ 014167 & China, Xizang, Motuo & MK135109 & MK805374 & Wang et al. (2020) \\
\hline P. niger & GP 1294 & China, Yunnan, Mengzi & MK135079 & MK805344 & Wang et al. (2020) \\
\hline P. niger & GP $3551=$ YBU 14251 & China, Yunnan, Mengzi & MK135080 & MK805345 & Wang et al. (2020) \\
\hline P. niger & GP $3552=$ YBU 14252 & China, Yunnan, Mengzi & MK135081 & MK805346 & Wang et al. (2020) \\
\hline P. niger & GP $3553=$ YBU 14253 & China, Yunnan, Mengzi & MK135082 & MK805347 & Wang et al. (2020) \\
\hline P. niger & GP $3588=$ YBU 14288 & China, Yunnan, Mengzi & MK135083 & MK805348 & Wang et al. (2020) \\
\hline P. niger & GP $4122=$ YBU 15100 & China, Yunnan, Kaiyuan & MK135084 & MK805349 & Wang et al. (2020) \\
\hline P. niger & GP $4123=$ YBU 15114 & China, Yunnan, Kaiyuan & MK135085 & MK805350 & Wang et al. (2020) \\
\hline P. nigriceps & CHS 656 = SYSr001222 & China, Yunnan, Gaoligongshan & MK201455 & - & Li et al. (2020) \\
\hline P. nuchalis & FK 2626 & Brunei, Belait & MZ603794 & U49311 & $\begin{array}{l}\text { This study, Kraus and } \\
\text { Brown (1998) }\end{array}$ \\
\hline P. stanleyi & GP 229 & China, Guangxi & MK135086 & MK805351 & Wang et al. (2020) \\
\hline P. stanleyi & GP $2343=$ YBU 12094 & China, Guizhou, Leishan & MK135087 & MK805352 & Wang et al. (2020) \\
\hline P. stanleyi & HM 2007-S001 & China, Guangxi, Guilin & JN230704 & JN230705 & Guo et al. (2011) \\
\hline P. temporalis sp. nov. & UNS 09992 & Vietnam, Lam Dong, Da Huoai & MZ603793 & MZ603792 & This study \\
\hline P. victorianus & CAS 235254 & Myanmar, Chin, Mt. Natmataung & MW438300 & MW438302 & Vogel et al. (2021) \\
\hline P. vindumi & CAS 248147 & Myanmar, Kachin, Chipwi, Lukpwi & MW287080 & MW287059 & Ding et al. (2020) \\
\hline P. xuelinensis & KIZ XL 1 & China, Yunnan, Lancang & MW436709 & - & Liu and Rao (2021) \\
\hline Xylophis captaini & BNHS 3376 & India, Kerala, Kottayam, Kannam & MK340914 & MK340912 & Deepak et al. (2018) \\
\hline$X$. perroteti & BNHS 3582 & India, Tamil Nadu, Nilgiri, Sholur & MN970042 & MN970046 & Deepak et al. (2020) \\
\hline
\end{tabular}

using raxml GUI 2.0 (Edler et al. 2021). The GTR $+\mathrm{I}+\mathrm{G}$ model was applied to the four partitions selected by PartitionFinder 2 (Lanfear et al. 2017) in a single analysis. Nodal support values were estimated by the thorough bootstrap with 1,000 pseudoreplicates. Nodes with pos- terior probabilities $\geq 0.95$ and bootstrap values $\geq 70$ were considered to be supported. Uncorrected pairwise $(p)$ distances were calculated using PAUP* version 4.0a165 (Swofford 2003). 


\section{Results}

\section{Phylogenetic analysis}

The dataset contained 1,971 aligned characters and 100 taxa. In the BI analysis, the standard deviation of split frequencies was 0.003490 among the four runs, and the Estimated Sample Sizes (ESS) of parameters were $\geq 2,248$. The Di Linh Plateau taxon was deeply nested within the P. carinatus complex (sensu Ding et al. 2020; Vogel et al. 2021) and recovered with strong support in both the BI and ML analyses to be the sister taxon of $P$. nuchalis, with a Bayesian posterior probability (PP) of 0.99 (Fig. 1) and a ML bootstrap (BS) value of 74 (Fig. 2). In turn, these two species were sister to a clade containing $P$. carinatus and P. menglaensis, with a Bayesian PP of 1.00 (Fig. 1) and a ML BS value of 100 (Fig. 2).

The Di Linh specimen had an uncorrected pairwise divergence in the coding region of cyt $b$ of $19.8 \%$ from $P$. nuchalis $(n=1), 19.8-20.2 \%$ from $P$. carinatus $(n=6)$, and $20.5-21.0 \%$ from $P$. menglaensis $(n=4)$. The Di Linh specimen had an uncorrected pairwise divergence in the coding region of ND4 of $17.9 \%$ from $P$. nuchalis $(n=1)$, $19.7-19.8 \%$ from $P$. carinatus $(n=4)$, and $19.6-19.8 \%$ from $P$. menglaensis $(n=4)$.

\section{Taxonomy}

\section{Pareas temporalis sp. nov.}

http://zoobank.org/DD72E44B-2EA4-4C34-AAB61799742733AF

Figures 3-5

Suggested Common Names. Di Linh Snail-eating Snake (English), Rắn hổ mây Di Linh (Vietnamese).

Holotype. UNS 09992 (field number LD25711), adult female, Vietnam, Lam Dong Province, Da Huoai District, Doan Ket Commune, $11.340370^{\circ} \mathrm{N}, 107.620561^{\circ} \mathrm{E}$, 496 m a.s.1., coll. 25 July 2020 by Duong T.T. Le and Thinh G. Tran.

Diagnosis. Pareas temporalis sp. nov. is distinguished from all other Pareas by having the combination of yellow-brown body colouration; hexagonal-shaped frontal, with lateral sides parallel to the body axis; $16-17$ temporals, with 4-5 anterior temporals; loreal and prefrontal not contacting eye; 2-3 preoculars; two suboculars; 2-3 postoculars; 8-9 supralabials; 8-9 infralabials (Fig. 4); 15-15-15 dorsal scale rows, all keeled (Fig. 3b), three vertebral scale rows enlarged; 191 (+1 preventral) ventrals, smooth; 92 subcaudals, all divided; undivided anal scale; two postocular stripes; and a solid dark brown vertebral stripe extending from rear of nuchal collar along the entire length of body and tail.
Description of the holotype. Adult female (Figs 3a, 5), SVL 426 mm; TaL 152 mm; TL 578 mm; TaL/TL 0.263. Body slender, compressed; head elongate, clearly distinct from neck; snout round in dorsal view; eye slightly enlarged, pupil vertical and slightly elliptical; rostral slightly visible in dorsal view; nasal scale single; two internasals, wider than long, contacting rostral, loreal, nasals, prefrontals; two prefrontals, large, not contacting eye; frontal hexagonal-shaped with lateral sides parallel to the body axis, frontal smaller than parietals; single loreal contacting nasal, internasal, prefrontal, preocular, $2^{\text {nd }}$ and $3^{\text {rd }}$ supralabial (left) or $2^{\text {nd }}$ supralabial (right), but not contacting eye; $1 / 1$ supraocular; $2 / 2$ suboculars; $2 / 3$ preoculars; $2 / 3$ postoculars; $9 / 8$ supralabial scales, $5^{\text {th }}, 6^{\text {th }}, 7^{\text {th }} / 4^{\text {th }}, 5^{\text {th }}$, $6^{\text {th }}$ below (not touching) eye, $9^{\text {th }} / 8^{\text {th }}$ longest; $16-17$ temporals $(4+3+4+6 / 5+3+4+4) ; 8 / 9$ infralabials, without mental groove; three pairs of chin shields, not equal in size, anterior pair of chin shields longer than broad, slightly longer than two posterior pairs; dorsal scales in 15-15-15 rows, all keeled without apical pits; three enlarged vertebral scale rows; 191 ventrals ( +1 preventral), all smooth; 92 subcaudals, all divided; undivided anal scale.

Colouration. In life, top of head light brown with dark brown spots. Sides of head with two postocular stripes: lower stripe extends from the postorbital to the $9^{\text {th }} / 8^{\text {th }}$ supralabial; upper stripe extends from the upper corner of the eye to the temporal area, then divides into two long stripes, with the upper arms meeting at the nape, while the lower arm extends to the corner of the jaw and sides of the neck before converging to form a black nuchal collar (collar six scales long at mid-dorsals). Ground colour of dorsum brown with dark-brown speckling and numerous irregular black cross-bands on lateral sides of body from neck to vent (64 bands on left and 62 bands on right), and a solid dark-brown vertebral stripe extending from the posterior end of the black nuchal collar along entire length of body and tail. Ventrals light brown with dark brown spots on lateral edges and middle of each scale, spotting weaker on chin shields. Ventral surface of tail dark brown. Colouration in preservative as in life, but with dorsum faded to yellowish brown.

Distribution and natural history. Pareas temporalis $\mathbf{s p .}$ nov. is currently only known by the holotype specimen from Da Huoai District, Lam Dong Province, southern Vietnam (Fig. 6). The sampling site is located near to the boundary of BinhThuan Province, and so it is likely that the new species is also found in forested areas of that province. The holotype was found at night (2100 hours) on a tree branch $1.5 \mathrm{~m}$ above the ground in disturbed mixed broadleaf and bamboo forest, where it occurred in sympatry with P. margaritophorus (e.g., UNS 09993). No further information is currently known on the biology of the new species.

Etymology. The specific epithet temporalis L. refers to the high number of temporal scales in the new species. 


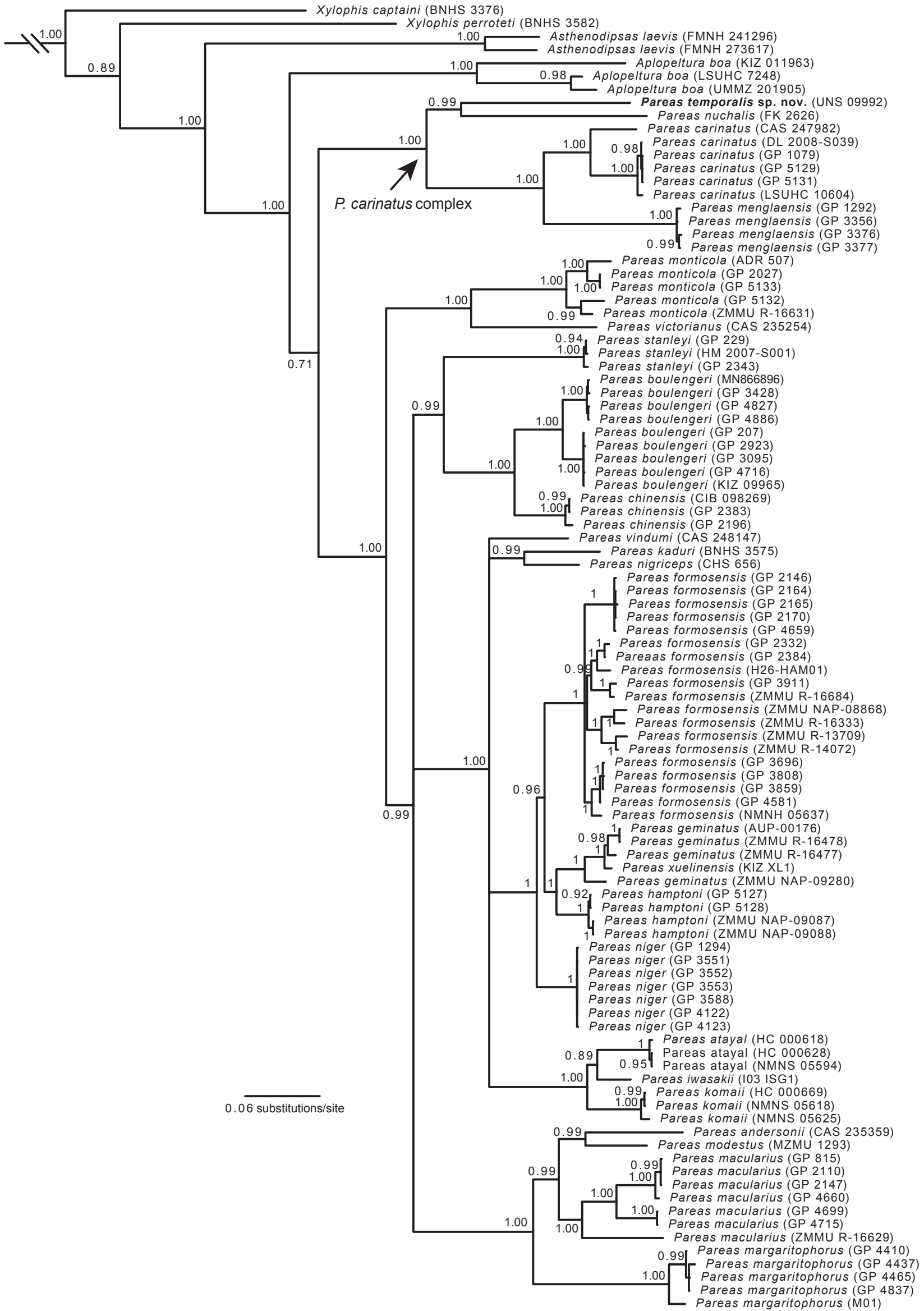

Figure 1. Fifty percent majority-rule consensus phylogram resulting from partitioned Bayesian analysis of 1,971 aligned characters of the mitochondrial cytochrome $b$ (cytb), NADH dehydrogenase subunit 4 (ND4), and flanking tRNA genes of pareid snakes. Trees were rooted with Achalinus rufescens (not shown). Numbers at nodes are Bayesian posterior probabilities. Sample information is provided in Table 1. 


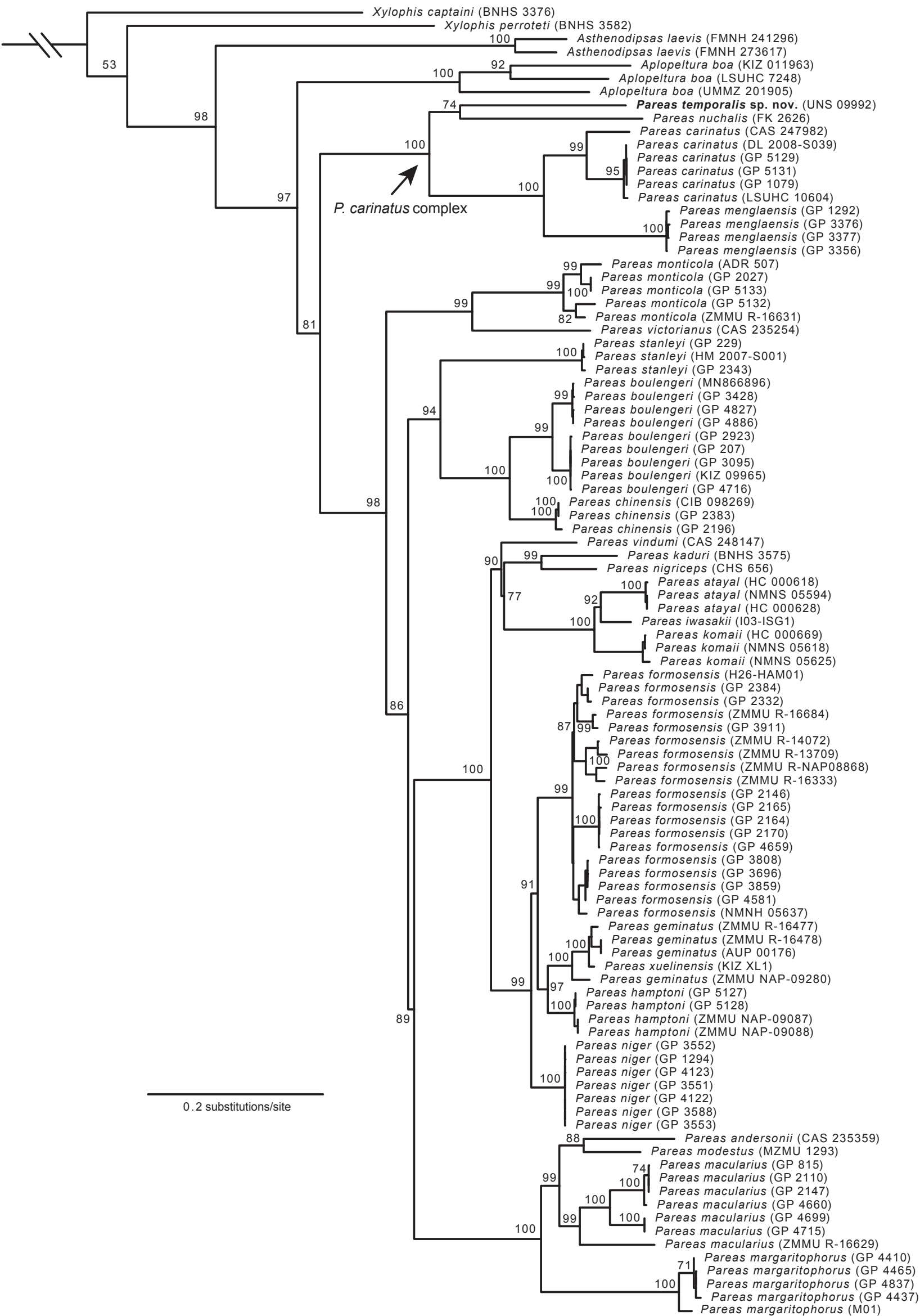

Figure 2. Maximum likelihood phylogeny based on a partitioned dataset containing 1,971 aligned characters of the mitochondrial cytochrome $b$ (cytb), NADH dehydrogenase subunit 4 (ND4), and flanking tRNA genes of pareid snakes. Trees were rooted with Achalinus rufescens (not shown). Numbers at nodes are bootstrap values based on 1,000 pseudoreplicates. Sample information is provided in Table 1. 

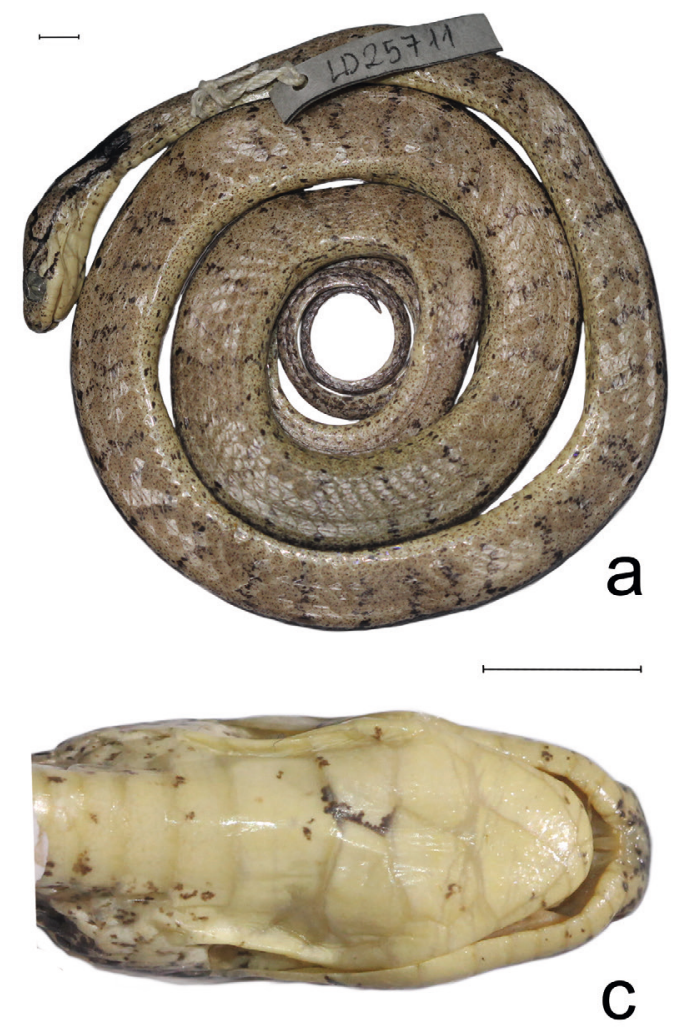

C

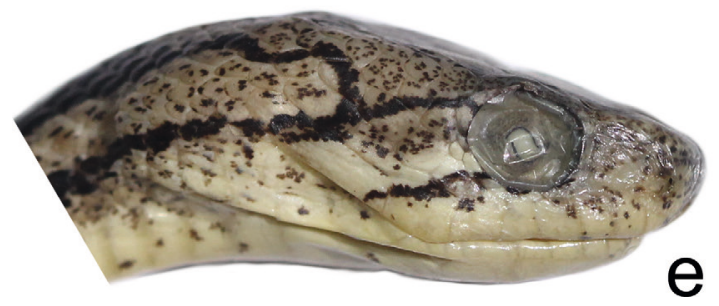

Figure 3. Holotype female (UNS 09992) of Pareas temporalis sp. nov. in preservative. a: whole body in lateral view; b: keeled dorsal scales at midbody; c: ventral view of head; d: dorsal view of head; e and f: lateral views of head. Scale bars: $5 \mathrm{~mm}$.
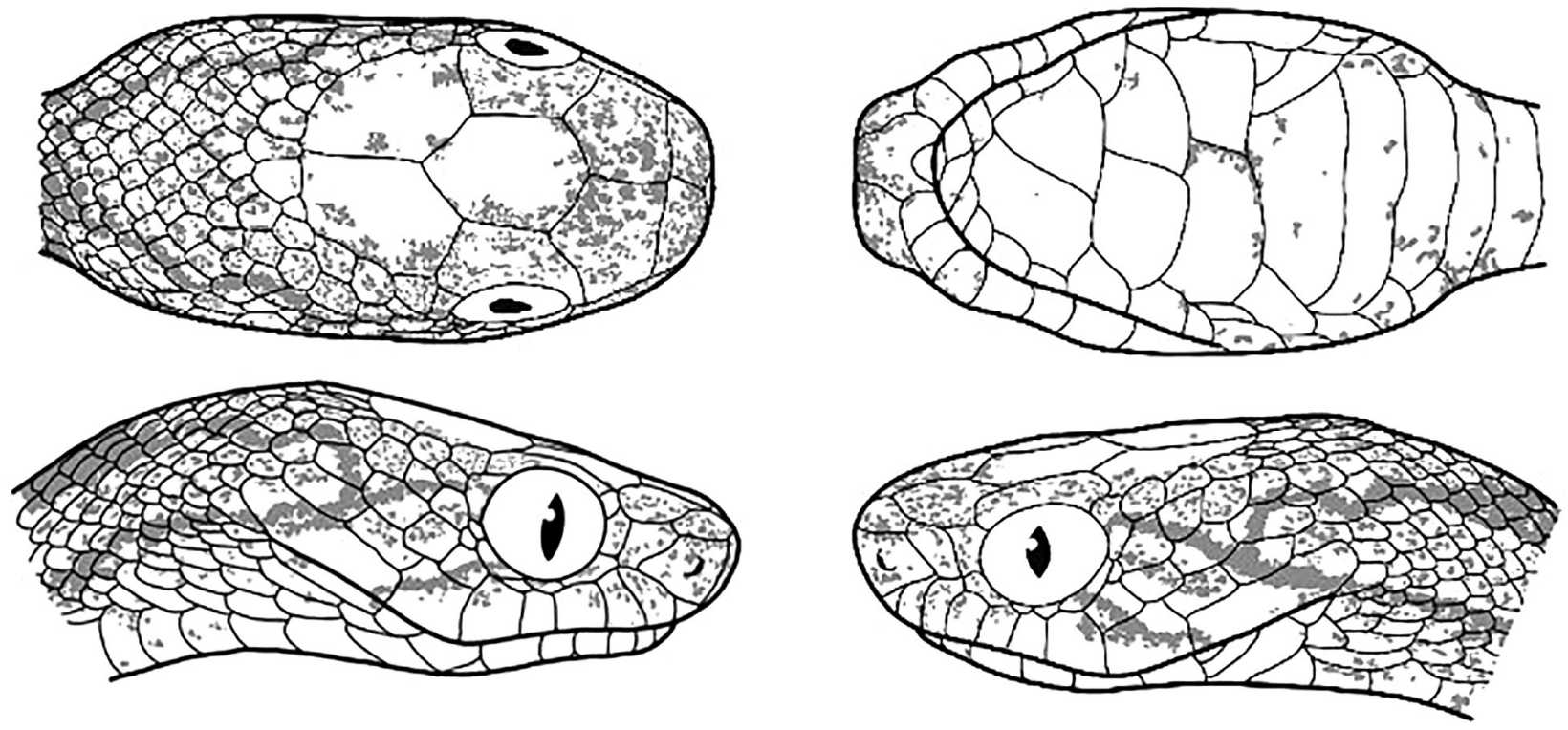

Figure 4. Head scalation of holotype female (UNS 09992) of Pareas temporalis sp. nov. in ventral, dorsal and lateral views. Illustration by Vo Ngoc Thinh. 

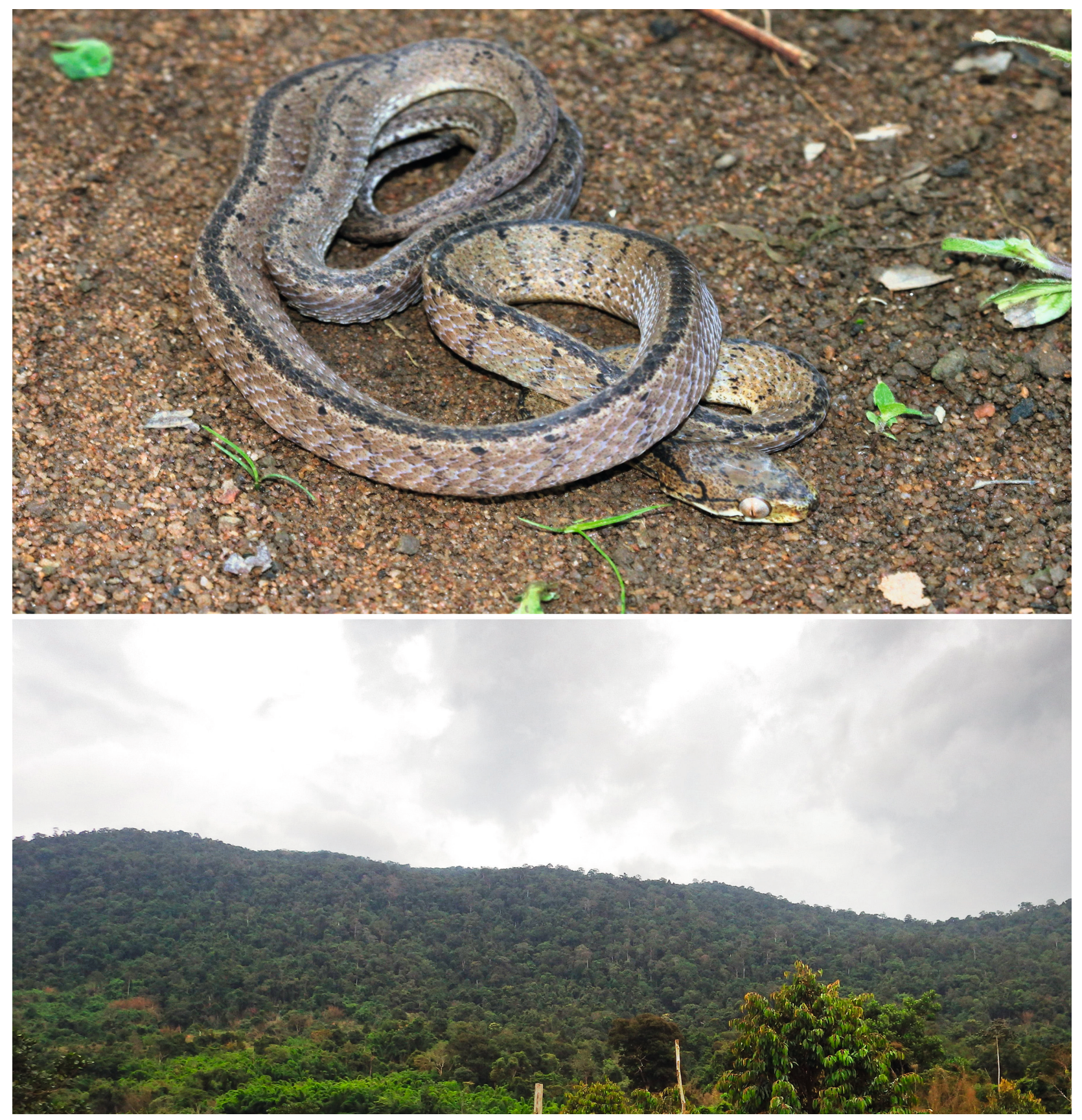

Figure 5. Holotype female (UNS 09992) of Pareas temporalis sp. nov. in life (above) and view of habitat at its type locality (below).

Comparisons. Pareas temporalis sp. nov. differs from P. margaritophorus, P. macularius, P. modestus Theobald, 1868 and $P$. andersonii (Boulenger, 1888) by having a light brown dorsum with irregular dark bands (vs. uniform grey to black to dark colouration, and with bicolored spots in P. margaritophorus, P. macularius and P. andersonii); prefrontal not contacting the eye (vs. contacting); fully keeled dorsal scale rows at midbody (vs. not fully keeled); three enlarged vertebral scales (vs. not enlarged); and frontal hexagonal with lateral sides parallel to body axis (vs. frontal subhexagonal with lateral sides converging posteriorly) (Ding et al. 2020; Suntrarachun et al. 2020; Vogel et al. 2020; Wang et al. 2020; Vogel et al. 2021).

Pareas temporalis sp. nov. differs from $P$. boulengeri (Angel, 1920), P. monticola (Cantor, 1839), P. stanleyi
(Boulenger, 1914), P. vindumi (Vogel, 2015), P. victorianus (Vogel, Nguyen, Zaw \& Poyarkov, 2021) and P. yunnanensis Vogt, 1922 by having the prefrontal not contacting the eye (vs. contacting); loreal not contacting the eye (vs. contacting); two suboculars (vs. 0-1 or suboculars fused with postoculars); 2-3 postoculars (vs 1-2 or postoculars fused with suboculars); fully keeled dorsal scale rows at midbody (vs. not fully keeled); 4-5 anterior temporals (vs. 1-2); and frontal hexagonal with lateral sides parallel to body axis (vs. frontal subhexagonal with lateral sides converging posteriorly) (Boulenger 1914; Guo and Deng 2009; Vogel 2015; Wang et al. 2020; Liu and Rao 2021; Vogel et al. 2021).

Pareas temporalis sp. nov. differs from $P$. geminatus Ding, Cheng, Suwannapoom, Nguyen, Poyarkov \& 
$105^{\circ} 0^{\prime} 0 " \mathrm{E}$

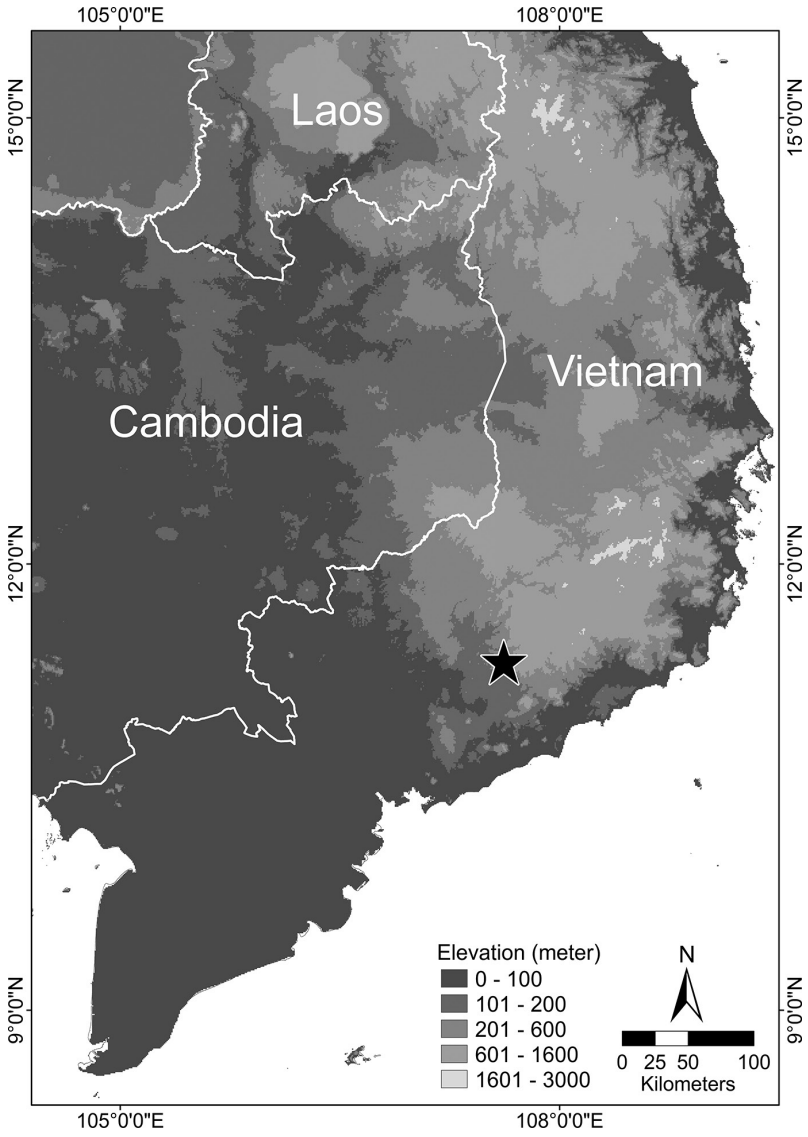

Figure 6. Holotype locality of Pareas temporalis sp. nov. (black star) in Lam Dong Province, Vietnam.

Vogel, 2020, P. atayal You, Poyarkov \& Lin, 2015, $P$. chinensis (Barbour, 1912), P. formosensis (Van Denburgh, 1909), P. hamptoni (Boulenger, 1905), P. iwasakii (Maki, 1937), P. komaii (Maki, 1931), P. niger Pope, 1928, P. xuelinensis Liu \& Rao, 2021, P. nigriceps Guo \& Deng, 2009 and P. kaduri Bhosale, Phansalkar, Sawant, Gowande, Patel \& Mirza, 2020 by having the prefrontal not contacting eye (vs. contacting, except in $P$. nigriceps); two suboculars (vs. one or suboculars fused with postoculars); 2-3 preoculars (vs. one); fully keeled dorsal scale rows at midbody (vs. not fully keeled); 4-5 anterior temporals (vs. 1-3); and frontal hexagonal with lateral sides parallel to body axis (vs. frontal subhexagonal with lateral sides converging posteriorly) (Guo and Deng 2009; You et al. 2015; Bhosale et al. 2020; Ding et al. 2020; Wang et al. 2020; Liu and Rao 2021; Vogel et al. 2021).

Pareas temporalis sp. nov. is most closely related (Figs 1-2) and morphologically similar to other members of the $P$. carinatus complex, consisting of $P$. carinatus (Wagler, 1830), P. nuchalis (Boulenger, 1900), and $P$. menglaensis Wang, Che, Liu, Li, Jin, Jiang, Shi \& Guo, 2020. Pareas temporalis sp. nov. differs from all three species by having 4-5 anterior temporals (vs. three); and a dark brown vertebral stripe on body and tail (vs. absent). Pareas temporalis sp. nov. further differs from $P$. carinatus by having 2-3 preoculars (vs. one); and all 15 dorsal scale rows keeled at midbody (vs. 0-11). Pareas temporalis sp. nov. further differs from $P$. nuchalis by having prefrontal not contacting eye (vs. contacting); 191 ventrals (vs. 195-220); and 92 subcaudals (vs. 102120). Pareas temporalis sp. nov. further differs from $P$. menglaensis by having 191 ventrals (vs. 176-177); 92 subcaudals (vs. 65-79); 2-3 postoculars (vs. one); 15 dorsal scale rows keeled at midbody (vs. 11); and two black postorbital stripes on lateral side of head (vs. one thin postorbital stripe extending from postocular to neck on lateral side of head) (Boulenger 1900; Malkmus et al. 2002; Guo and Deng 2009; Stuebing et al. 2014; Ding et al. 2020; Wang et al. 2020; Vogel et al. 2021).

\section{Discussion}

Recent phylogenetic analyses of Pareas have revealed that the genus contains two major clades, the $P$. carinatus complex/group (Ding et al. 2020; Vogel et al. 2021; referred to "Pareas I" by Guo et al. 2011) consisting of $P$. carinatus, $P$. nuchalis, and $P$. menglaensis, and a second clade consisting of all other congeners (Guo et al. 2011; Ding et al. 2020; Vogel et al. 2020; Wang et al. 2020; Vogel et al. 2021). Guo et al. (2011) suggested that the $P$. carinatus complex (as "Pareas I") may represent a distinct genus from other "Pareas" on the basis of large $p$-distances, their lack of statistical support for the monophyly of Pareas with respect to Aplopeltura and Asthenodipsas, and morphological diagnosability in having at least three anterior temporals, the frontal scale hexagonal with the lateral sides parallel to the body axis, and the anterior pair of chin shields broader than long. Our molecular phylogenetic analyses found $P$. temporalis sp. nov. to be a fourth member of the $P$. carinatus complex, and consistent with Guo et al.'s (2011) morphological diagnosis of the clade.

The description of Pareas temporalis sp. nov. from southern Vietnam brings the total number of recognized Pareas species to 25, of which seven occur in Vietnam (P. carinatus, P. formosensis, P. hamptoni, P. macularius, $P$. margaritophorus, $P$. monticola, and $P$. temporalis sp. nov.) (Nguyen et al. 2009; Ding et al. 2020). However, with only one specimen collected, information on the precise distribution, natural history, ecology, population status and conservation of the new species is unknown, as with many other Pareas species (see IUCN 2021; Uetz et al. 2021). We suggest that the new species be considered Data Deficient (DD) following IUCN's Red List categories (IUCN 2021). However, it should be noted that the type locality of $P$. temporalis sp. nov. consists of disturbed forest on the Di Linh Plateau where no protected areas have been established. Remaining natural forests in the area are under high degree of threat from conversion to agricultural lands and commercial crops. Further surveys for $P$. temporalis $\mathbf{s p . ~ n o v . , ~ a n d ~ t h e ~ p o s s i b l e ~ e x i s - ~}$ tence of other endemic lineages on the Di Linh Plateau, are warranted. 


\section{An updated key to the $P$. carinatus complex is provided below:}

1 Prefrontal in contact with eye, $\geq 102$ subcaudals (Borneo) ......................................................P. nuchalis

- Prefrontal not in contact with eye, $<102$ subcaudals...................................................................................2

2 All dorsal scale rows keeled at midbody, 4-5 anterior temporals, anterior pair of chin shields longer than broad ...

P. temporalis sp. nov.

- Some dorsal scale rows keeled at midbody, three anterior temporals, anterior pair of chin shields broader than long.

3 One preocular, a black line from eye to nape, and another from behind eye to angle of mouth P. carinatus

- Two preoculars, a thin postorbital stripe extending from postocular to neck P. menglaensis

\section{Funding}

This research was supported by funding from The Nagao Natural Environment Foundation (NEF).

\section{Competing interests}

The authors have declared that no competing interests exist.

\section{Acknowledgements}

The People's Committee of Doan Ket Commune, Da Huoai District, Lam Dong Province, Vietnam, kindly facilitated surveys and issued a specimen collection permit (permit number 0025/GGT-KHTN). Ka Giang assisted with fieldwork. Pham Manh Hung assisted with DNA sequencing. Vo Ngoc Thinh illustrated Figure 4. Fred Kraus, Greg Schneider, and Ulmar Grafe helped to determine the disposition of the P. nuchalis specimen bearing field number FK 2626, and Dan Rabosky and Sonal Singhal generously donated the cyt $b$ sequence from that sample that they obtained as bycatch in a phylogenomic analysis that is underway. Ke Jiang provided additional locality data for the $P$. carinatus samples used in Wang et al. (2020). For all this assistance we are most grateful.

\section{References}

Angel MF (1920) Liste de reptiles récémment déterminés et entrés dans les collections et description d'une nouvelle espèce du genre Amblycephalus. Bulletin du Muséum National d'Histoire Naturelle 2: 112-114.

Arèvalo E, Davis SK, Sites JW (1994) Mitochondrial DNA sequence divergence and phylogenetic relationships among eight chromosome races of the Sceloporus grammicuscomplex (Phrynosomatidae) in central Mexico. Systematic Biology 43(3): 387-418. https://doi. org/10.1093/sysbio/43.3.387

Barbour T (1912) Some Chinese vertebrates: amphibia and reptilia Memoirs of the Museum of Comparative Zoölogy 40(4): 125-136.

Bhosale H, Phansalkar P, Sawant M, Gowande G, Patel H, Mirza ZA (2021) A new species of snail-eating snakes of the genus Pareas Wagler, 1830 (Reptilia: Serpentes) from eastern Himalayas, India. European Journal of Taxonomy 729: 54-73. https://doi.org/10.5852/ ejt.2020.729.1191

Bocourt F (1866) Notes sur les reptiles. Les batraciens et les poissons recueillis pendant un voyage dans le Royaume de Siam. Nouvelles Archives du Museum d'Histoire Naturelle De Paris 2: 4-20.
Boulenger GA (1888) An account of the reptilia obtained in Burma, north of Tenasserim, by M. L. Fea, of the Genova Civic Museum. Annali del Museo civico di storia naturale di Genova 2(6): 593-604.

Boulenger GA (1900) Descriptions of new reptiles and batrachians from Borneo. In: Proceedings of the Zoological Society of London. Blackwell Publishing Ltd, Oxford, 69(2): 182-187.

Boulenger GA (1905) Descriptions of two new snakes from upper Burma. Journal of the Bombay Natural History Society 16: 235-236.

Boulenger GA (1914) Descriptions of new species of snakes in the collection of the British Museum. Annals and Magazine of Natural History 14(84): 482-485. https://doi.org/10.1080/00222931408693606

Burbrink FT, Lawson R, Slowinski JB (2000) Mitochondrial DNA phylogeography of the polytypic North American rat snake (Elaphe obsoleta): a critique of the subspecies concept. Evolution 54(6): 2107-2118. https://doi.org/10.1111/j.0014-3820.2000.tb01253.x

Cantor TE (1839) Spicilegium serpentium indicorum. Part I. In: Proceedings of the Zoological Society of London, 1839: 31-34.

Deepak V, Ruane S, Gower DJ (2018) A new subfamily of fossorial colubroid snakes from the Western Ghats of peninsular India. Journal of Natural History 52(45-46): 2919-2934. https://doi.org/10.1080/0 0222933.2018 .1557756

Deepak V, Narayanan S, Das S, Rajkumar KP, Easa PS, Sreejith KA, Gower DJ (2020) Description of a new species of Xylophis Beddome, 1878 (Serpentes: Pareidae: Xylophiinae) from the Western Ghats, India. Zootaxa 4755: 231-250. https://doi.org/10.11646/zootaxa.4755.2.2

Ding L, Chen ZN, Suwannapoom C, Nguyen TV, Poyarkov NA, Vogel G (2020) A new species of the Pareas hamptoni complex (Squamata: Serpentes: Pareidae) from the Golden Triangle. Taprobanica 9(2): 174-193. https://doi.org/10.47605/tapro.v9i2.230

Edler D, Klein J, Antonelli A, Silvestro D (2021) raxml GUI 2.0: A graphical interface and toolkit for phylogenetic analyses using RAxML. Methods in Ecology and Evolution 12: 373-377. https://doi. org/10.1111/2041-210X.13512

Figueroa A, McKelvy AD, Grismer LL, Bell CD, Lailvaux SP (2016) A species-level phylogeny of extant snakes with description of a new colubrid subfamily and genus. PLoS ONE 11: e0161070. https://doi. org/10.1371/journal.pone.0161070

Grossmann W, Tillack F (2003) On the taxonomic status of Asthenodipsas tropidonotus (Van Lidth de Jeude, 1923) and Pareas vertebralis (Boulenger, 1900) (Serpentes: Colubridae: Pareatinae). Russian Journal of Herpetology 10(3): 175-190.

Guo KJ, Deng XJ (2009) A new species of Pareas (Serpentes: Colubridae: Pareatinae) from the Gaoligong Mountains, southwestern China. Zootaxa 2008: 53-60. https://doi.org/10.11646/zootaxa.2008.1.5

Guo Y, Wu Y, He S, Shi H, Zhao E (2011) Systematics and molecular phylogenetics of Asian snail-eating snakes (Pareatidae). Zootaxa 3001: 57-64. https://doi.org/10.11646/zootaxa.3001.1.4 
Hauser S (2017) On the validity of Pareas macularius Theobald, 1868 (Squamata: Pareidae) as a species distinct from Pareas margaritophorus (Jan in Bocourt, 1866). Tropical Natural History 17(1): $25-52$.

Huang QY (2004) Pareas macularius Theobald, 1868 should be a junior synonym of Pareas margaritophorus (Jan, 1866). Sichuan Journal of Zoology 23(3): 207-208.

Huang R, Peng L, Yang D, Yong Z, Huang S (2020) Mitochondrial genome of the Boulenger's Slug-eating snake Pareas boulengeri (Serpentes: Pareidae). Mitochondrial DNA Part B 5: 3179-3180. https:// doi.org/10.1080/23802359.2020.1804471

International Union for the Conservation of Nature (IUCN) Species Survival Commission (SSC) Amphibian Specialist Group (2017) The IUCN Red List of Threatened Species. https://www.iucnredlist. org

Katoh K, Standley DM (2013) MAFFT multiple sequence slignment software version 7: improvements in performance and stability. Molecular Biology and Evolution 30: 772-780. https://doi.org/10.1093/ molbev/mst010

Kraus F, Brown WM (1998) Phylogenetic relationships of colubroid snakes based on mitochondrial DNA sequences. Zoological Journal of the Linnean Society 122: 455-487. https://doi.org/10.1111/j.1096-3642.1998.tb02159.x

Lanfear R, Frandsen PB, Wright AM, Senfeld T, Calcott B (2017) PartitionFinder 2: new methods for selecting partitioned models of evolution for molecular and morphological phylogenetic analyses. Molecular Biology and Evolution 34: 772-773. https://doi.org/10.1093/ molbev/msw260

Li J-N, Liang D, Wang Y-Y, Guo P, Huang S, Zhang P (2020) A largescale systematic framework of Chinese snakes based on a unified multilocus marker system. Molecular Phylogenetics and Evolution 148: 106807. https://doi.org/10.1016/j.ympev.2020.106807

Liu S, Rao D (2021) A new species of the genus Pareas (Squamata, Pareidae) from Yunnan, China. ZooKeys 1011: 121-138. https://doi. org/10.3897/zookeys.1011.59029

Loredo AI, Wood PL, Quah ESH, Anuar S, Greer LF, Ahmad N, Grismer LL (2013) Cryptic speciation within Asthenodipsas vertebralis (Boulenger, 1900) (Squamata: Pareatidae), the description of a new species from Peninsular Malaysia, and the resurrection of $A$. tropidonotus (Lidth de Jue, 1923) from Sumatra: an integrative taxonomic analysis. Zootaxa 3664: 505-524. https://doi.org/10.11646/ zootaxa.3664.4.5

Maki M (1931) Monograph of the snakes of Japan. Dai-ichi Shobo, Tokyo, $240 \mathrm{pp}$.

Maki M (1937) A new subspecies, Amblycephalus formosensis iwasakii, belonging to Amblycephalidae from Ishigaki-jima. Transactions, Natural History Society of Formosa 27: 217-218.

Malkmus R, Manthey U, Vogel G, Hoffmann P, Kosuch J (2002) Amphibians and Reptiles of Mount Kinabalu (North Borneo). A.R.G. Ganter Verlag Kommanditgesellschaft, Ruggel, 424 pp.

Miller MA, Pfeiffer W, Schwartz T (2010) Creating the CIPRES Science Gateway for inference of large phylogenetic trees. In: Proceedings of the Gateway Computing Environments Workshop (GCE), 14 Nov. 2010, New Orleans, LA, pp. 1-8. https://doi.org/10.1109/ GCE.2010.5676129

Nguyen VS, Ho TC, Nguyen QT (2009) Herpetofauna of Vietnam. Edition Chimaira, Frankfurt, 768 pp.

Pope CH (1928) Four new snakes and a new lizard from South China. American Museum Novitates 325: 1-4.
Pyron RA, Burbrink FT, Wiens JJ (2013) A phylogeny and revised classification of Squamata, including 4161 species of lizards and snakes. BMC Evolutionary Biology 13: 93. https://doi.org/10.1186/14712148-13-93

Quah ESH, Grismer LL, Lim KKP, Shahrul AMS, Imbun PY (2019) A taxonomic reappraisal of the Smooth Slug Snake Asthenodipsas laevis (Boie, 1827) (Squamata: Pareidae) in Borneo with the description of two new species. Zootaxa 4646(3): 501-526. https:// doi.org/10.11646/zootaxa.4646.3.4

Rambaut A, Drummond AJ, Xie D, Baele G, Suchard MA (2018) Posterior summarization in Bayesian phylogenetics using Tracer 1.7. Systematic Biology 67: 901-904. https://doi.org/10.1093/sysbio/ syy032

Rao DQ, Yang DT (1992) Phylogenetic systematics of Pareatinae (Serpentes) of Southeastern Asia and adjacent islands with relationship between it and the geology changes. Acta Zoologica Sinica 38: 139-150.

Ronquist F, Teslenko M, Van der Mark P, Ayres DL, Darling A, Höhna S, Larget B, Liu L, Suchard MA, Huelsenbeck JP (2012) MrBayes 3.2: efficient Bayesian phylogenetic inference and model choice across a large model space. Systematic Biology 61: 1-4. https://doi. org/10.1093/sysbio/sys029

Stuebing RB, Inger RF, Lardner B (2014) A field guide to the snakes of Borneo. Natural History Publication, Borneo, Kota Kinabalu, 310 pp.

Suntrarachun S, Chanhome L, Hauser S, Sumontha M, Kanya K (2020) Molecular phylogenetic support to the resurrection of Pareas macularius from the synonymy of Pareas margaritophorus (Squamata: Pareidae). Tropical Natural History 20(2): 182-190.

Swofford DL (2003) PAUP*: Phylogenetic Analysis Using Parsimony *(and Other Methods). Sinauer Associates, Sunderland, Massachusetts, $142 \mathrm{pp}$.

Theobald W (1868) Catalogue of the reptiles of British Birma, embracing the provinces of Pegu, Martaban, and Tenasserim; with descriptions of new or little-known species. Zoological Journal of the Linnean Society 10: 4-67. https://doi.org/10.1111/j.1096-3642.1868. tb02007.x

Uetz P, Freed P, Hošek J (2021) The Reptile Database. http://www.reptile-database.org [Accessed on 25 May 2021].

van Denburgh J (1909) New and previously unrecorded species of reptiles and amphibians from the island of Formosa. Proceedings of the California Academy of Sciences 3: 49-56.

Vogel G (2015) A new montane species of the genus Pareas Wagler, 1830 (Squamata: Pareatidae) from northern Myanmar. Taprobanica 7(1): $1-7$.

Vogel G, Nguyen TV, Lalremsanga HT, Biakzuala L, Hrima V, Poyarkov NA (2020) Taxonomic reassessment of the Pareas margaritophorus-macularius species complex (Squamata, Pareidae). Vertebrate Zoology 70(4): 547-569. https://doi.org/10.26049/VZ704-2020-02

Vogel G, Nguyen TV, Zaw T, Poyarkov NA (2021) A new species of the Pareas monticola complex (Squamata: Serpentes: Pareidae) from Chin Mountains with additions to the Pareas fauna of Myanmar. Journal of Natural History 54(39-40): 2577-612. https://doi.org/10. 1080/00222933.2020.1856953

Vogt T (1922) Zur reptilien-und amphibienfauna Südchinas. Archiv für Naturgeschichte 88(10): 135-146.

Wang P, Che J, Liu Q, Li K, Jin JQ, Jiang K, Shi L, Guo P (2020) A revised taxonomy of Asian snail-eating snakes Pareas (Squamata, 
Pareidae): evidence from morphological comparison and molecular phylogeny. ZooKeys 939: 45-64. https://doi.org/10.3897/zookeys.939.49309

You CW, Poyarkov NA, Lin SM (2015) Diversity of the snail-eating snakes Pareas (Serpentes, Pareatidae) from Taiwan. Zoologica Scripta 44(4): 349-361. https://doi.org/10.1111/zsc.12111
Zhang Y, Yang D-C, Peng L-F, Jin A, Duan S, Huang S (2017) Complete mitochondrial genome of the Rufous burrowing snake, Achalinus rufescens (Reptilia: Xenodermatidae). Mitochondrial DNA Part B 2 : 419-420. https://doi.org/10.1080/23802359.2017.1347842 